\title{
Déficit de satisfacción de necesidades en usuarios de drogas solicitantes de tratamiento
}

\author{
Raúl García-Aurrecoechea *; Rogelio Díaz-Guerrero **; María Elena Medina-Mora *** \\ * Departamento de Investigación Clínica y Epidemiológica, Centros de Integración Juvenil \\ ** (1918-2004) Facultad de Psicología, Universidad Nacional Autónoma de México \\ ** División de Investigaciones Epidemiológicas y Sociales, Instituto Nacional de Psiquiatría "Ramón de la Fuente" \\ Enviar correspondencia a: \\ Raúl García Aurrecoechea. Subjefe de Investigación Clínica y Epidemiológica. Centros de Integración Juvenil. \\ Tlaxcala 208, Colonia Condesa. C. P. 06100 México, D. F.. e-mail: raurre@hotmail.com
}

\section{RESUMEN}

Como parte de una línea de investigación pionera en el ámbito de las adicciones y la salud mental, enfocada en operacionalizar las implicaciones clínicas de la teoría de la motivación de Maslow (1954/1970), que retroalimente sustantivamente las estrategias de tratamiento y prevención de la adicción del consumo de drogas y sus trastornos asociados, se somete a prueba el constructo de psicopatogénesis de esta teoría por medio de un diseño transversal de cuatro muestras independientes en el cual se evalúa el grado de satisfacción de 16 necesidades deficitarias de muestras intencionales de adolescentes y adultos jóvenes: una de éstas integrada por estudiantes y trabajadores no usuarios de drogas ilegales $(n=151)$ y tres más compuestas por usuarios actuales de marihuana $(n=47)$, de cocaína $(n=47)$ y de ambas sustancias $(n=50)$, los cuales fueron captados entre solicitantes de tratamiento a Centros de Integración Juvenil. Los análisis estadísticos comparativos y predictivos proporcionaron validez al constructo de psicopatogénesis de la teoría de la motivación de Maslow y se señala: 1) la utilidad del desarrollo de técnicas e instrumentos de tratamiento orientados a cubrir el déficit de satisfacción de las necesidades de salud, tranquilidad, orden, seguridad emocional, justicia en la familia, amor, amistad, respeto, cariño, poder, dominar, éxito y dinero y 2) el beneficio de elaborar estrategias que se orienten a mantener satisfechas las necesidades deficitarias de salud, tranquilidad, cariño, respeto y éxito en el campo la prevención del consumo actual de drogas como la cocaína o la marihuana.

Palabras clave: cocaína, marihuana, prevención, tratamiento, Maslow.

\section{ABSTRACT}

As part of a pioneer investigation line on the field of addiction and mental health centred on the operationalization of clinical implications of the motivational theory of Maslow (1954/1970) and feedback treatment and prevention strategies of drug use and its associated disturbances, it is tested the psycho-pathogenesis construct of this theory by means of a cross sectional design of four independent samples, on which it is explored the satisfaction degree of 16 deficitary needs on intentional samples of adolescents and young adults: Three samples of actual users of marihuana $(n=47)$, cocaine $(n=47)$ and both substances $(n=50)$, that were gotten between treatment solicitors and a sample of students and workers non illicit drug users $(n=151)$. The comparative and predictive statistical analysis provide validity to the psycho-pathogenesis construct of the theory of motivation of Maslow, and its stand out: 1) The potential utility for the treatment of the development of techniques and instruments oriented to cover the deficit of satisfaction of the needs of health, tranquillity, order, emotional security, family justice, love, friendship, respect, tenderness, power, domination, success and money and; 2) The importance for the prevention of the actual consumption of drugs as cocaine or marihuana of the development of strategies focused to keep satisfied the needs of health, tranquillity, affection, respect and success.

Key words: cocaine, marihuana, prevention, treatment, Maslow.
$\mathbf{L}$ a teoría de la motivación de Maslow (1954/1970) tiene un fundamento psicosocial que plantea que las necesidades deficitarias (fisiológicas, seguridad, afectivas y estima) deben ser satisfechas desde fuera por otros distintos del ser, a partir de las relaciones que se sostiene con ellos, existiendo una tenden- cia a satisfacer primero los niveles más básicos y a medida que se va logrando, cobran mayor importancia los niveles más elevados.

La teoría cuenta también con implicaciones clínicas, puesto que propone que un bajo grado de satis- 
facción de algunas de estas necesidades conduce a la generación de distintos tipos de trastornos mentales (psicopatogénesis), los cuales irían desapareciendo en la medida en que disminuyera el déficit de satisfacción existente.

Un problema particular que se relaciona con la deficiencia de éxito, lo constituye el complejo de Jonás (Maslow, 1990), el cual se debe a un temor a lograr las potencialidades más elevadas, dado que éstas hacen conflicto con la interiorización que el sujeto ha hecho de pobres expectativas que otros tienen de él y que se hacen manifiestas en relaciones depauperadas de determinadas satisfacciones psicosociales.

En México, desde los años sesenta, se han llevado a cabo estudios para identificar el grado de satisfacción de necesidades culturalmente relevantes, acordes con la pirámide de Maslow (Díaz-Guerrero y Díaz-Loving, 2001). La operacionalización de indicadores de motivación la llevó a cabo Díaz-Guerrero en 1967, por medio de la exploración del grado de satisfacción de 20 necesidades relevantes en adolescentes mexicanos, a partir de una escala ordinal pictográfica con los valores: 1=muy insatisfecho, 2=insatisfecho, 3=ni satisfecho ni insatisfecho, 4=satisfecho y 5=muy satisfecho (DíazGuerrero, Moreno-Cedillos y Díaz-Loving, 1995),

Estudios posteriores identificaron el grado de satisfacción de 30 necesidades culturalmente relevantes para los mexicanos, las cuales representaron los cinco niveles de la pirámide de Maslow (Díaz-Guerrero y Díaz-Loving, 2001). Un análisis factorial confirmatorio (García Aurrecoechea y Gracia, 2004a) y uno exploratorio (Mendoza, 2004) aportan validez de constructo de esta estructura.

También se ha reportado, a partir de la comparación de distintos estudios realizados con población urbana mexicana, que la escala del grado de satisfacción de las necesidades (Díaz-Guerrero y Díaz-Loving, 2001) presenta un comportamiento confiable y consistente a través del tiempo (Díaz-Guerrero et al., 1995).

Este estudio forma parte de una línea de investigación más amplia -pionera en el ámbito de las adicciones y de la salud mental- enfocada en operacionalizar las implicaciones clínicas de la teoría de la motivación de Maslow (1954/1970b) y en retroalimentar estrategias de tratamiento y prevención del consumo de drogas.

Estudios previos de esta línea han aportado sustento empírico a los constructos de psicopatogénesis y del Complejo de Jonás de la teoría de la motivación de Maslow y han identificado, en el contexto de esta teoría, indicadores de riesgo y protección del consumo de drogas y sus trastornos relacionados (García-Aurrecoechea, Díaz-Guerrero, Reyes-Lagunes, Medina-Mora, Andrade-Palos y Reidl, 2006), de los cuales ha derivado una escala multifactorial de riesgo y protección de estas problemáticas (García-Aurrecoechea, RodríguezKuri y Córdova, 2006).

Los estudios mencionados también han sido útiles en el desarrollo de estrategias de tratamiento multifactoriales en la intervención de la problemática del consumo de drogas y sus trastornos asociados (García-Aurrecoechea, Gracia, Gutiérrez-López, Balanzario y Guerrero-Huesca, 2005; García-Aurrecoechea, Fukushima, Gracia, Jiménez-Silvestre y Cielo, 2006).

Este estudio en particular tuvo como objetivo someter a prueba las hipótesis potencialmente universales de la teoría de la motivación de Maslow con relación a la psicopatogénesis y en particular en el caso del consumo de drogas (Maslow, 1954/1970a y 1954/1970b) en muestras de adolescentes y adultos jóvenes del sexo masculino, por medio de la evaluación del grado de satisfacción de sus necesidades deficitarias y esperó: a) que se presentaran menores niveles de satisfacción de las necesidades deficitarias de los grupos de consumidores de marihuana, cocaína o ambas drogas y b) que la satisfacción de determinadas necesidades deficitarias cumpliera una función potencialmente protectora del consumo de marihuana, de cocaína o de ambas drogas. De la primera hipótesis se obtuvieron recomendaciones para el tratamiento y de la segunda recomendaciones para la prevención.

\section{MÉTODO}

Para comprobar estas hipótesis se evaluó, a través de un diseño transversal ex-post-facto, el grado de satisfacción de un conjunto de necesidades deficitarias en cuatro muestras independientes de mediano tamaño del sector poblacional más afectado por la problemática del consumo de drogas: los adolescentes y adultos jóvenes del sexo masculino, las cuales fueron elegidas en forma intencional: tres de ellas estuvieron integradas por grupos de usuarios de distintas drogas ilícitas y una más conformada por estudiantes y trabajadores no consumidores de drogas ilícitas.

Debido a la dificultad para ubicar los usuarios de drogas en escenarios naturales, se determinó la obtención de muestras intencionales entre pacientes solicitantes de tratamiento del consumo de drogas de 17 unidades de Centros de Integración Juvenil (CIJ) de la Cd. de México, con el apoyo de sus terapeutas, los cuales administraron cuestionarios auto-aplicables y verificaron 
que los participantes no reportaran el uso actual de solventes inhalables, heroína o metanfetamina.

A su vez, la muestra de no usuarios de drogas fue obtenida entre estudiantes (de secundaria, preparatoria y profesional) y trabajadores que no presentaran consumo actual de drogas de curso ilegal (en caso de haberlas consumido alguna vez, que hubiera sido de forma experimental: cinco veces o menos). La aplicación de cuestionarios a los no usuarios de drogas corrió por cuenta de promotores preventivos de ClJ, realizándose aplicaciones grupales en el caso de los estudiantes. La información fue recabada entre finales de 2002 y principios de 2003.

El instrumento formó parte de otro más extenso que indagó otras variables psicosociales, habiendo sido utilizados para este estudio una sección de identificación sociodemográfica y del patrón de consumo de sustancias (adoptado del formato de Entrevista Inicial utilizado en estudios epidemiológicos del consumo de drogas en ClJ por Castillo-Franco y Gutiérrez-López, 2003) y un cuestionario de 16 reactivos acerca del grado de satisfacción de necesidades deficitarias, basado en el Inventario de Necesidades y Valores desarrollado por Díaz-Guerrero y Díaz-Loving (2001), el cual obtuvo una adecuada confiabilidad en su aplicación (Alfa de Cronbach $=.88$ ), habiendo sido evaluados el grado de satisfacción de las siguientes necesidades:

- Fisiológicas: salud, comer, dormir.

- Seguridad: tranquilidad, orden, seguridad emocional, justicia en la familia.

- Afectivas: amor, amistad, respeto, cariño.

- Estima: reconocimiento, poder, dominar, éxito, dinero.

Las opciones de respuesta del grado de satisfacción se presentaron en un formato pictográfico, con los siguientes valores: $1=$ muy insatisfecho, $2=$ insatisfecho, 3= ni satisfecho ni insatisfecho, 4= satisfecho y $5=$ muy satisfecho.

Los análisis estadísticos se dividieron en dos fases, en la Primera Fase se realizaron 16 pruebas de ANOVA de una sola vía entre los grupos (para identificar potenciales necesidades de tratamiento) y en la Segunda Fase -considerando como variables independientes las variables que mostraron diferencias estadísticamente significativas en la Primera Fas- se elaboraron tres pruebas multivariadas de Regresiones Logísticas Binarias entre los no usuarios y cada uno de los grupos de consumidores, en las cuales se consideró a los grados de satisfacción de las diferentes necesidades deficitarias como potenciales factores de protección al codificar al no consumo de drogas como uno y como cero el caso del uso de drogas. Los datos fueron procesados por medio del programa estadístico SPSS v.10.

\section{RESULTADOS}

En la Primera Fase, las pruebas de ANOVA de una sola vía encontraron diferencias estadísticamente significativas en 14 de las 16 necesidades deficitarias, apareciendo carencias de satisfacción en todos los casos entre los grupos de consumidores frente a los no usuarios de drogas, con algunas diferencias entre los grupos de usuarios.

Con respecto a las necesidades fisiológicas, en el grado de satisfacción de la necesidad de salud el grupo de no usuarios de drogas lo ubicó claramente en satisfecho, mientras que los grupos de policonsumidores y consumidores de cocaína lo ubicaron más cercano a medianamente satisfecho. La prueba Tukey también reveló una diferencia estadística entre los consumidores de marihuana y los policonsumidores, reafirmándose la deficiencia de salud en este último grupo. No se presentaron deficiencias de satisfacción en los usuarios de marihuana. (Tabla 1).

Con respecto a las necesidades de seguridad, en el grado de satisfacción de la tranquilidad el grupo de no usuarios de drogas la reportó cercano a satisfecho frente a los consumidores de cocaína (inferior a medianamente satisfecha) y en menor grado frente a los de marihuana (un poco arriba de medianamente satisfecha), siendo también significativa la deficiencia de satisfacción de los consumidores de cocaína ante los poliusuarios y los usuarios de marihuana (Tabla 1).

Al grado de satisfacción de la necesidad de orden el grupo de no usuarios de drogas lo ubicó más cercano a satisfecho que los usuarios de cocaína y los policonsumidores, los cuales lo consideraron medianamente satisfecho, sin observarse diferencias significativas entre los grupos de no usuarios y usuarios de marihuana (Tabla 1).

Con respecto al grado de satisfacción de la seguridad emocional, los no consumidores lo evaluaron satisfecho, a diferencia de los tres grupos de consumidores, quienes lo evaluaron medianamente satisfecho. (Tabla 1).

Al grado de satisfacción de la justicia en la familia los no usuarios de drogas lo consideraron satisfecho, a diferencia de los grupos de consumidores, mas cercanos a un grado medianamente satisfecho (Tabla 1). 
Tabla 1. ANOVA de una sola vía del Grado de Satisfacción de las necesidades Fisiológicas y de Seguridad $(n=295)$

\begin{tabular}{|c|c|c|c|c|c|c|c|c|}
\hline Necesidad & Grupos & $\mathbf{n}$ & media & $D E$ & $\boldsymbol{F}$ & gl & $p$ & Post hoc* \\
\hline \multicolumn{9}{|c|}{ Necesidades Fisiológicas (2=insatisfecho; 4=satisfecho) } \\
\hline \multirow{4}{*}{ Salud } & No consumo & 148 & 4.09 & 0.78 & \multirow{4}{*}{11.9} & \multirow{4}{*}{3} & \multirow{4}{*}{.000} & \multirow{4}{*}{$\begin{array}{c}n>p, c \\
m>p\end{array}$} \\
\hline & Marihuana & 46 & 3.78 & 0.84 & & & & \\
\hline & Cocaína & 46 & 3.63 & 0.9 & & & & \\
\hline & Policonsumo & 50 & 3.34 & 0.87 & & & & \\
\hline \multirow{4}{*}{ Comer } & No consumo & 147 & 4.13 & 0.75 & \multirow{4}{*}{1.2} & \multirow{4}{*}{3} & \multirow{4}{*}{ ns } & \\
\hline & Marihuana & 46 & 4.07 & 0.80 & & & & \\
\hline & Cocaína & 46 & 4.00 & 0.82 & & & & \\
\hline & Policonsumo & 49 & 3.90 & 0.85 & & & & \\
\hline \multirow{4}{*}{ Dormir } & No consumo & 147 & 3.78 & 0.88 & \multirow{4}{*}{0.8} & \multirow{4}{*}{3} & \multirow{4}{*}{ ns } & \\
\hline & Marihuana & 46 & 3.78 & 0.94 & & & & \\
\hline & Cocaína & 46 & 3.63 & 1.00 & & & & \\
\hline & Policonsumo & 50 & 3.58 & 0.97 & & & & \\
\hline \multicolumn{9}{|c|}{ Necesidades de Seguridad (2=insatisfecho; 4=satisfecho) } \\
\hline \multirow{4}{*}{ Tranquilidad } & No consumo & 148 & 3.73 & 0.85 & \multirow{4}{*}{17.43} & \multirow{4}{*}{3} & \multirow{4}{*}{.000} & \multirow{4}{*}{$\begin{array}{c}\mathrm{n}>\mathrm{c}, \mathrm{m} \\
\mathrm{m}>\mathrm{c} \\
\mathrm{p}>\mathrm{c}\end{array}$} \\
\hline & Marihuana & 46 & 3.24 & 1.06 & & & & \\
\hline & Cocaína & 46 & 2.63 & 0.95 & & & & \\
\hline & Policonsumo & 50 & 3.32 & 0.98 & & & & \\
\hline \multirow{4}{*}{ Orden } & No consumo & 148 & 3.68 & 0.79 & \multirow{4}{*}{9.23} & \multirow{4}{*}{3} & \multirow{4}{*}{.000} & \multirow[t]{4}{*}{$n>c, p$} \\
\hline & Marihuana & 46 & 3.26 & 1.12 & & & & \\
\hline & Cocaína & 46 & 2.87 & 1.05 & & & & \\
\hline & Policonsumo & 50 & 3.16 & 1.15 & & & & \\
\hline \multirow{4}{*}{$\begin{array}{l}\text { Seguridad } \\
\text { Emocional }\end{array}$} & No consumo & 147 & 3.92 & 0.87 & \multirow{4}{*}{17.65} & \multirow{4}{*}{3} & \multirow{4}{*}{.000} & $\mathrm{n}>\mathrm{c}, \mathrm{p}, \mathrm{m}$ \\
\hline & Marihuana & 46 & 3.26 & 1.12 & & & & \\
\hline & Cocaína & 46 & 2.87 & 1.05 & & & & \\
\hline & Policonsumo & 50 & 3.16 & 1.15 & & & & \\
\hline & No consumo & 151 & 3.95 & 0.98 & & & & $n>c, p, m$ \\
\hline Justicia en la & Marihuana & 46 & 3.39 & 1 & & & & \\
\hline Familia & Cocaína & 46 & 3.07 & 1.04 & 10.05 & 3 & . & \\
\hline & Policonsumo & 50 & 3.16 & 1.11 & & & & \\
\hline
\end{tabular}

* Tukey ( $n$ = no consumo; $m$ = marihuana; c = cocaína; $p$ = policonsumo)

$\mathrm{ns}=$ no significativo 
Tabla 2. ANOVA de una sola vía del Grado de Satisfacción de las necesidades Afectivas

\begin{tabular}{|c|c|c|c|c|c|c|c|c|}
\hline Necesidad & Grupos & $\mathbf{n}$ & media & $D E$ & $\boldsymbol{F}$ & gl & $\boldsymbol{p}$ & Post hoc* \\
\hline \multicolumn{9}{|c|}{ Necesidades Afectivas (2=insatisfecho; 4=satisfecho) } \\
\hline \multirow{4}{*}{ Amor } & No consumo & 151 & 3.90 & 1.13 & \multirow{4}{*}{3.20} & \multirow{4}{*}{3} & \multirow{4}{*}{.024} & \\
\hline & Marihuana & 46 & 3.52 & 1.19 & & & & \\
\hline & Cocaína & 46 & 3.46 & 1.17 & & & & \\
\hline & Policonsumo & 50 & 3.50 & 1.04 & & & & \\
\hline \multirow{4}{*}{ Amistad } & No consumo & 151 & 4.26 & 0.85 & \multirow{4}{*}{12.19} & \multirow{4}{*}{3} & \multirow{4}{*}{.000} & \multirow[t]{4}{*}{$n>c, m, p$} \\
\hline & Marihuana & 46 & 3.63 & 1.1 & & & & \\
\hline & Cocaína & 46 & 3.41 & 1.24 & & & & \\
\hline & Policonsumo & 50 & 3.66 & 1.1 & & & & \\
\hline \multirow{4}{*}{ Respeto } & No consumo & 147 & 4.03 & 0.76 & \multirow{4}{*}{10.28} & \multirow{4}{*}{3} & \multirow{4}{*}{.000} & \multirow[t]{4}{*}{$n>m, p, c$} \\
\hline & Marihuana & 46 & 3.46 & 0.89 & & & & \\
\hline & Cocaína & 46 & 3.48 & 0.96 & & & & \\
\hline & Policonsumo & 50 & 3.46 & 1.01 & & & & \\
\hline \multirow{4}{*}{ Cariño } & No consumo & 147 & 4.24 & 0.8 & \multirow{4}{*}{12.44} & \multirow{4}{*}{3} & \multirow{4}{*}{.000} & \multirow[t]{4}{*}{$n>m, p, c$} \\
\hline & Marihuana & 46 & 3.63 & 1.06 & & & & \\
\hline & Cocaína & 46 & 3.72 & 0.91 & & & & \\
\hline & Policonsumo & 50 & 3.48 & 1.01 & & & & \\
\hline
\end{tabular}

Tukey ( $\mathrm{n}=$ no consumo; $\mathrm{m}=$ marihuana; $\mathrm{c}=$ cocaína; $\mathrm{p}=$ policonsumo) $\mathrm{ns}=$ no significativo

Con respecto a las necesidades Afectivas, en el grado de satisfacción de la necesidad de amor se observaron diferencias significativas y se aprecia que los no usuarios lo ubicaron cercano a satisfecho, mientras que los tres grupos de usuarios de drogas lo consideraron más cercano a medianamente satisfecho, aunque las pruebas post hoc no identificaron específicamente los grupos en que se presentan las diferencias (Tabla 2).

Se observaron diferencias en el grado de satisfacción de la necesidad de amistad, encontrándose más que satisfecha entre los no usuarios de drogas, mientras que los tres grupos de consumidores lo ubicaron intermedio entre medianamente satisfecho y satisfecho (Tabla 2).

En el grado de satisfacción de la necesidad de respeto las diferencias se presentan entre los no usuarios de drogas (quienes lo valoraron como satisfecho), y los tres grupos de consumidores (que lo ubicaron más cercano a medianamente satisfecho) (Tabla 2).

Al grado de satisfacción de cariño los no usuarios de drogas lo encontraron un poco arriba de satisfecho, a diferencia de los tres grupos de consumidores, que lo ubicaron entre medianamente satisfecho y satisfecho (Tabla 2).
Con respecto a las necesidades de Estima, se detectaron diferencias en el grado de satisfacción de la necesidad de reconocimiento entre los no usuarios de drogas (más cercanos a satisfecho) y los consumidores de cocaína y los poliusuarios (cercanos a medianamente satisfecho), sin apreciarse diferencias estadísticas con los usuarios de marihuana (Tabla 3).

Al grado de satisfacción del poder la prueba $F$ reportó diferencias entre los grupos, aunque las pruebas post hoc no identificaron los grupos que las presentaron. No obstante, puede observarse que los no consumidores de drogas lo ubicaron más cercano a satisfecho y que los grupos de usuarios lo ubicaron mas cercano a medianamente satisfecho (Tabla 3).

De forma similar a la anterior, en grado de satisfacción de la necesidad de dominar el estadístico $F$ reportó diferencias entre los grupos, pero las pruebas post hoc no lograron precisar entre cuales grupos se presentaron las diferencias. Sin embargo se aprecian mayores puntajes en los no usuarios de drogas (lo ubicaron entre medianamente satisfecho y satisfecho) que entre los grupos de consumidores (más cercanos a medianamente satisfecho), sobre todo en el caso de los policonsumidores, (Tabla 3). 
Tabla 3. ANOVA de una sola vía del Grado de Satisfacción de las necesidades de Estima

\begin{tabular}{|c|c|c|c|c|c|c|c|c|}
\hline Necesidad & Grupos & $\mathbf{n}$ & media & $D E$ & $\boldsymbol{F}$ & $g l$ & $p$ & Post hoc* \\
\hline \multicolumn{9}{|c|}{ Necesidades de Estima (2=insatisfecho; 4=satisfecho) } \\
\hline \multirow{4}{*}{ Reconocimiento } & No consumo & 150 & 3.75 & 0.82 & \multirow{4}{*}{7.70} & \multirow{4}{*}{3} & \multirow{4}{*}{.000} & \multirow[t]{4}{*}{$n>c, p$} \\
\hline & Marihuana & 45 & 3.40 & 0.89 & & & & \\
\hline & Cocaína & 46 & 3.17 & 1.02 & & & & \\
\hline & Policonsumo & 50 & 3.18 & 1.12 & & & & \\
\hline \multirow{4}{*}{ Poder } & No consumo & 150 & 3.62 & 0.83 & \multirow{4}{*}{4.27} & \multirow{4}{*}{3} & \multirow{4}{*}{.006} & \\
\hline & Marihuana & 46 & 3.37 & 0.74 & & & & \\
\hline & Cocaína & 46 & 3.24 & 0.92 & & & & \\
\hline & Policonsumo & 50 & 3.24 & 0.82 & & & & \\
\hline \multirow{4}{*}{ Dominar } & No consumo & 150 & 3.52 & 0.91 & \multirow{4}{*}{2.62} & \multirow{4}{*}{3} & \multirow{4}{*}{.051} & \\
\hline & Marihuana & 46 & 3.37 & 0.68 & & & & \\
\hline & Cocaína & 45 & 3.4 & 0.86 & & & & \\
\hline & Policonsumo & 49 & 3.12 & 0.9 & & & & \\
\hline \multirow{4}{*}{ Éxito } & No consumo & 150 & 3.77 & 0.91 & \multirow{4}{*}{16.67} & \multirow{4}{*}{3} & \multirow{4}{*}{.000} & \multirow[t]{4}{*}{$n>c, m, p$} \\
\hline & Marihuana & 46 & 2.96 & 1.21 & & & & \\
\hline & Cocaína & 46 & 2.80 & 1.11 & & & & \\
\hline & Policonsumo & 50 & 3.02 & 1.08 & & & & \\
\hline \multirow{4}{*}{ Dinero } & No consumo & 151 & 3.49 & 0.96 & \multirow{4}{*}{8.03} & \multirow{4}{*}{3} & \multirow{4}{*}{.000} & \multirow[t]{4}{*}{$n>c, p$} \\
\hline & Marihuana & 46 & 3.04 & 1.05 & & & & \\
\hline & Cocaína & 46 & 2.78 & 1.01 & & & & \\
\hline & Policonsumo & 50 & 3.02 & 0.96 & & & & \\
\hline
\end{tabular}

* Tukey ( $n$ = no consumo; $m$ = marihuana; $c$ = cocaína; $p=$ policonsumo)

En el grado de satisfacción de la necesidad de éxito las diferencias se presentaron entre los no usuarios de drogas (que lo ubicaron cercano a satisfecho) y los tres grupos de usuarios, quienes lo consideraron medianamente satisfecho (Tabla 3).

Finalmente, al grado de satisfacción de la necesidad de dinero los no usuarios de drogas lo ubicaron entre medianamente satisfecho y satisfecho, presentando diferencias con los consumidores de cocaína y los policonsumidores (ubicados alrededor de medianamente satisfecho), sin que se observaran diferencias con los usuarios de marihuana en esta variable (Tabla 3).

En la Segunda Fase las pruebas de regresiones logísticas binarias arrojaron tres robustos modelos, uno para cada tipo de consumidor (cocaína, marihuana o ambas drogas), en éstas se consideró a los grados de satisfacción de las necesidades deficitarias como potenciales indicadores de protección y se utilizó el método "adelante condicional" para introducir dichas variables independientes, respectivamente.

Para el caso del análisis de los policonsumidores de marihuana y cocaína frente a los no consumidores, se encontró un modelo en que la satisfacción de las necesidades de salud (Wald=14.325, $g l=1, p<.01$, $\operatorname{Exp}(B)=2.341$ ) y cariño ( $W a l d=10.810, g l=1, p<.01$, $\operatorname{Exp}(B)=1.946)$ protegen contra el policonsumo de estas sustancias, la primera 2.3 veces y la segunda dos veces. El modelo predijo correctamente la pertenencia a los grupos en el $79.3 \%$ de los casos.

Para el caso del análisis de los usuarios de cocaína frente a los no consumidores, se encontró que la satisfacción de las necesidades de tranquilidad ( $W a l d=18.784, g l=1, p<.01, \operatorname{Exp}(B)=2.942)$ y de éxito $($ Wald $=6.707, g l=1, p \leq .01, \operatorname{Exp}(B)=1.824)$ protegen contra el consumo de cocaína, la primera 2.9 veces y la segunda 1.8 veces. En este caso el modelo pre- 
dijo correctamente la pertenencia a los grupos en el $84.1 \%$ de los casos.

Para el caso del análisis de los usuarios de marihuana frente a los no consumidores, se encontró que la satisfacción de las necesidades de éxito ( Wald=6.935, $g l=1, p<.01, \operatorname{Exp}(B)=1.687$ ) y de respeto (Wald=5.576, $g l=1, p<.05, \operatorname{Exp}(B)=1.78)$ protegen contra el consumo de marihuana, la primera 1.7 veces y la segunda 1.8 veces. En este caso el modelo predijo correctamente la pertenencia a los grupos en el $79.4 \%$ de los casos.

\section{DISCUSIÓN}

Los resultados son acordes con las hipótesis potencialmente universales de la teoría de la motivación de Maslow (1954/1970a y 1954/1970b) y su relación con los trastornos mentales para el caso del consumo de cocaína, marihuana y ambas drogas, siendo éstos relevantes para la cultura mexicana (Díaz-Guerrero, Moreno-Cedillos y Díaz-Loving, 1995).
En la Primera Fase se identificaron menores grados de satisfacción en 14 necesidades deficitarias frente a los no consumidores, con ciertas diferencias entre los grupos de usuarios, aportándose al tratamiento la posibilidad de abordar factores motivacionales relacionados con el consumo actual de sustancias, útiles para mejorar la adherencia terapéutica y orientar el tratamiento, que de acuerdo con la teoría, debieran tratarse desde los niveles más básicos hacia los más elevados, es decir de la base a la cúspide de la pirámide (Figura 1).

En la Segunda Fase se identificaron tres distintos pares de deficiencias de satisfacción de necesidades asociadas con la predicción, los cuales se consideraron como factores de protección del consumo, que pudieran ser útiles para la prevención del uso de estas sustancias, siendo también de utilidad en el tratamiento, puesto que pueden prevenir las recaídas. Por lo cual, resultaría importante promover en la sociedad el valor de satisfacer estas necesidades y analizar más a fondo los factores de riesgo asociados que impiden su satisfacción (Figura 2).

Figura 1. Pirámide de necesidades deficitarias de tratamiento del consumo de drogas*

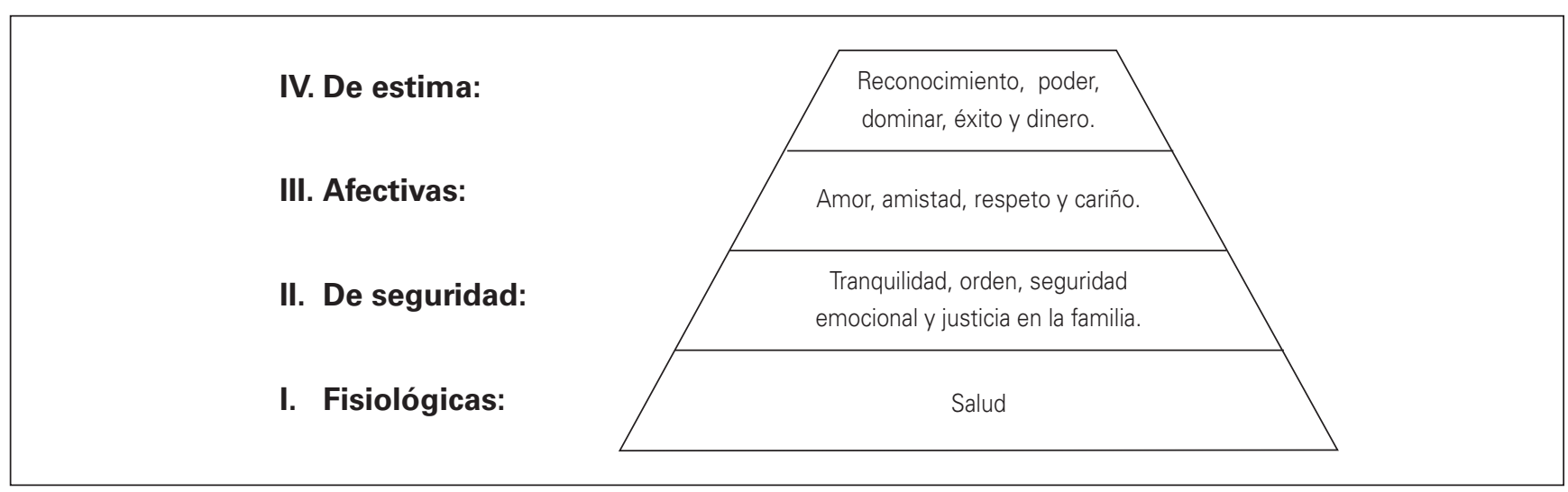

* Para tratamiento del consumo de cocaína, marihuana o ambas drogas

Figura 2. Pirámide de necesidades deficitarias protectoras del consumo de drogas*

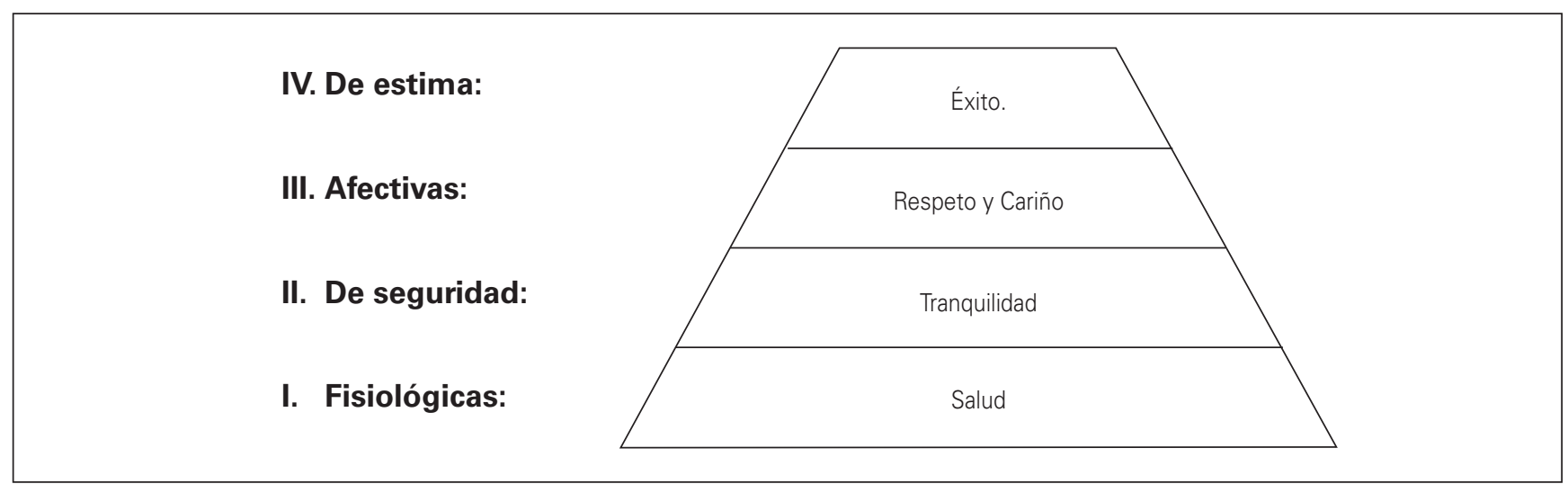

\footnotetext{
* Para prevención del consumo de cocaína, marihuana o ambas drogas
} 
De esta forma, como prevención del policonsumo, se destaca la importancia de tener satisfechas las necesidades de salud y de cariño, pudiendo estas necesidades relacionarse en el sentido de que tener cariño por alguien se puede manifestar con cuidar y enseñarle a cuidar su salud.

Como prevención del consumo de cocaína cobra importancia la satisfacción de las necesidades de tranquilidad y de éxito, con mayor peso la primera y surge la pregunta: ¿qué ocupa el lugar de la tranquilidad en estos usuarios?

Como prevención del consumo de marihuana aparece relevante, también, la satisfacción de la necesidad de éxito y la del respeto, la pregunta en este caso es ¿qué factores evitan la existencia del respeto?

Se destaca la motivación de los dos últimos grupos por tener éxito, lo cual puede estar mediado por el complejo de Jonás (Maslow, 1990), en donde se manifiesta una motivación latente por "salir adelante" y por "ser alguien en la vida".

De tal manera, se considera que esta información inicia una veta importante en el estudio de los trastornos mentales, la cual, aporta indicadores y factores asociados con el mono y poli-consumo de marihuana y/o cocaína, útiles para el tratamiento y prevención. Por lo que, por una parte, podrían explorarse posibles deficiencias de satisfacción de necesidades en otros grupos de usuarios y en otros tipos de trastornos y por otra parte, podría ampliarse el espectro de necesidades estudiadas y su grado de satisfacción.

Por otra parte, como limitaciones del estudio se debe de considerar que las muestras de consumidores correspondieron a solicitantes de tratamiento, por lo que las generalizaciones a todo tipo de usuarios de drogas debieran hacerse con cautela. Asimismo, el diseño transversal y ex-post-facto presenta limitaciones en las conclusiones derivadas de los análisis predictivos, dado que se basa en datos sincrónicos.

Por lo cual, sería conveniente replicar y ampliar el análisis del déficit de satisfacción de necesidades en muestras más amplias y con diseños más poderosos, aunque con la evidencia obtenida se cuenta con elementos sustantivos para realizar evaluaciones piloto de la aplicación de estos resultados en el tratamiento y la prevención del consumo de sustancias ilegales.

\section{REFERENCIAS}

Castillo-Franco, P. I., Gutiérrez-López, A. D. y Gracia, S. E. (2003). Consumo de Drogas entre Pacientes que Ingresaron a Tratamiento en Centros de Integración Juvenil en 2002. Informe de investigación 03-01. México: Centros de Integración Juvenil, A.C.

Díaz-Guerrero, R., Alonso-Dávila, K. y Rivera-Aragón, S. (2001). Hacia el origen psicológico de los valores. Una replica metodológica. Revista de Psicología Social y Personalidad, 17 (2), 103-112.

Díaz-Guerrero, R. y Díaz-Loving, R. (2001). El origen psicológico de los valores. Revista de Psicología Social y Personalidad, 17 (2), 91-101.

Díaz-Guerrero, R., Moreno-Cedillos y Díaz-Loving, R. (1995). Un eslabón perdido en la investigación sobre valores y su persistencia. Revista de Psicología Social y Personalidad, 9 (1), 1-10.

García-Aurrecoechea R., Díaz-Guerrero R., Reyes-Lagunes I., Medina-Mora, M. E., Andrade-Palos, P. y Reidl, L. (2006). Indicadores psicosociales de motivación del consumo de marihuana y/o cocaína. Adicciones, 18(4): 387-398, 2006.

García-Aurrecoechea R., Fukushima E. A., Gracia S. E., Jiménez-Silvestre K, Cielo D. B. (2006). Evaluación formativa de un programa de tratamiento teóricamente sustentado. Segunda fase: prueba de instrumentos para la evaluación de un programa de Tratamiento para Usuarios de Estimulantes (TUE). México: Centros de Integración Juvenil. Informe de Investigación 06-12.

García-Aurrecoechea, V. R. y Gracia, S. E. (2004a, octubre). Adaptación del modelo de la pirámide de necesidades de Maslow en jóvenes de la Ciudad de México. Sesión de cartel presentada en el décimo congreso de la Asociación Mexicana de Psicología Social (AMEPSO), Cd. Obregón, Sonora, México.

García-Aurrecoechea, V. R. y Gracia, S. E. (2004b). Grado de satisfacción de necesidades y consumo actual de mariguana y/o cocaína en jóvenes varones solicitantes de tratamiento. México: Centros de Integración Juvenil, Dirección de Investigación y Enseñanza, Subdirección de Investigación. Informe de Investigación 04-10.

García Aurrecoechea, V. R., Gracia, S. E., Gutiérrez López, A. D., Balanzario, M. C. y Guerrero, A. (2005). Evaluación formativa de un programa de tratamiento teóricamente sustentado. Primera fase: evaluación de necesidades de tratamiento breve individual del trastorno de abuso de cocaína/crack. México: Centros de Integración Juvenil. Informe de Investigación 05-17.

García-Aurrecoechea, R., Rodríguez-Kuri, S. E., Córdova, A. (2006). Validación de Escalas de Riesgo y Protección de Trastornos del Afecto y Consumo de Drogas. México: Centros de Integración Juvenil. Informe de Investigación 06-17.

Maslow, A. (1954/1970a) A theory of human motivation. En A. Maslow, Motivation and personality (pp. 35-57). New York and London: Harper and Row Publishers.

Maslow, A. (1954/1970b) Psycho-pathogenesis and the theory of threat. En A. Maslow, Motivation and personality (pp. 105-115). New York and London: Harper and Row Publishers.

Maslow, A. (1990). La neurosis como deficiencia del desarrollo personal. En A. Maslow, La amplitud potencial de la naturaleza humana (pp. 39-53). México: Trillas.

Mendoza, A. R. (2004). Necesidades y valores: comparación entre dos grupos de trabajadores de una Universidad pública. Tesis de maestría no publicada. México: Universidad del Estado de Yucatán. 